\title{
Utilização de psicofármacos por pacientes odontológicos em Minas Gerais, Brasil
}

\author{
Mauro Henrique Nogueira Guimarães de Abreu, ${ }^{1}$ \\ Francisco de Assis Acúrcio ${ }^{2}$ e Vera Lúcia Silva Resende ${ }^{3}$
}

RESUMO A utilização de medicamentos no Brasil, inclusive dos psicofármacos, tem sido considerada exacerbada e indiscriminada. Mesmo assim, é reduzido o número de trabalhos sobre a utilização destes medicamentos, principalmente entre usuários de serviços odontológicos. O estudo teve como objetivo a verificação da prevalência de consumo de psicofármacos, nos períodos de 15 dias e de 12 meses que antecederam o estudo, entre os usuários das clinicas integradas de atenção primária da Faculdade de Odontologia, Universidade Federal de Minas Gerais, Brasil. A coleta de dados utilizou formulários de entrevistas aplicados a todos os indivíduos maiores de 12 anos, atendidos nestas clínicas durante o mês de junho de 1997. O consumo de psicofármacos foi de $4 \%$ e 10\%, respectivamente, para os 15 dias e 12 meses que antecederam o estudo. Os ansioliticos foram os mais consumidos nos 12 meses antes do estudo, totalizando aproximadamente $40 \%$ do consumo global. O grupo de indivíduos com idade até 23 anos (mediana da idade) apresentou um consumo menor do que o grupo com idade superior a 23 anos $(\mathrm{P}<$ $0,01)$. Houve uma associação significativa $(\mathrm{P}<0,05)$ entre o sexo feminino e o consumo destas drogas, bem como entre a profissão de dona de casa e o consumo $(\mathrm{P}<0,03)$. Os usuários que relataram serem casados ou amasiados (união estável) apresentaram maior consumo de psicofármacos quando comparados com os solteiros, viúvos ou separados (união instável) $(\mathrm{P}<$ 0,03). Não houve associação entre a utilização de psicofármacos e a escolaridade. Apesar da importância da informação a respeito do uso de psicofármacos para o diagnóstico e planejamento integral em odontologia, apenas $40 \%$ dos alunos das clinicas integradas relataram ter anotado este dado em ficha clínica. Tal fato pode indicar que o ensino de Odontologia está sendo falho neste aspecto, devendo, então, ser repensada a importância dada à questão dos medicamentos durante a formação dos cirurgiões-dentistas.

A utilização de medicamentos no Brasil tem sido considerada exacer-

\footnotetext{
1 Universidade Federal de Minas Gerais, Faculdade de Odontologia, Programa de Pós-Graduação em Odontologia, Belo Horizonte, MG, Brasil. Correspondência e pedidos de separatas devem ser enviados para este autor para o seguinte endereço: Rua Três Corações 300, apartamento 802A, Prado, CEP 30480-110, Belo Horizonte, Minas Gerais, Brasil. Telefone: +55-31-334-0146.

2 Universidade Federal de Minas Gerais, Faculdade de Farmácia, Departamento de Farmácia Social.

3 Universidade Federal de Minas Gerais, Faculdade de Odontologia, Departamento de Odontologia Restauradora. E-mail: silres@dedalus.lcc.ufmg.br
}

bada e indiscriminada. As causas deste padrão de consumo podem ser relacionadas ao pouco controle exercido pelo estado sobre a produção e comercialização dos medicamentos, à propaganda da indústria farmacêutica, ao baixo nível de renda da população brasileira frente aos altos custos dos serviços médicos e à eficácia de muitos medicamentos em relação a determinadas doenças (1-5).

Os psicofármacos, ou drogas psicotrópicas, são aqueles que interferem primariamente em funções do sistema nervoso central. Neste grupo de medicamentos estão incluídos os ansiolíticos, os antidepressivos, os antipsicóticos e os antiepiléticos $(6,7)$.

Os ansiolíticos são medicamentos que têm como objetivo a redução da ansiedade, sendo a classe dos benzodiazepínicos (BZD) a mais utilizada. Os representantes dos BZD são o clordiazepóxido, diazepam, lorazepam, oxazepam, midazolam e triazolam, dentre outros (7-9). Em odontologia, estas drogas são indicadas para a diminuição da ansiedade do paciente 
pouco cooperador para o tratamento ambulatorial, bem como na pré-medicação da anestesia geral (9-11). Os antidepressivos tricíclicos, cujo protótipo é a imipramina, são muito utilizados na terapêutica medicamentosa da depressão endógena ou maior.

Os compostos antipsicóticos ou neurolépticos são drogas usadas no tratamento das psicoses, tendo como protótipo a clorpromazina e o haloperidol. A psicose mais afetada pelo uso de antipsicóticos é a esquizofrenia $(7,8)$. As drogas antiepiléticas são utilizadas no tratamento da epilepsia. Como representantes deste grupo de drogas temse a fenitoína, o fenobarbital, a primidona e a carbamazepina, entre outros $(12,13)$.

O consumo dos psicofármacos é considerado alto em todo o mundo. Nos Estados Unidos, 8 milhões de pessoas apresentam alguma doença mental e, destas, aproximadamente 2 milhões fazem uso de farmacoterapia (14). Estudos mostram que de $10 \%$ a $20 \%$ das receitas passadas nos Estados Unidos são de psicofármacos $(8,13)$, sendo que o diazepam foi o fármaco mais prescrito nos Estados Unidos em 1978 e 1979 (14). Mais recentemente, é verificada uma mudança no padrão de prescrição de psicofármacos nos Estados Unidos, com maior utilização de antidepressivos (15).

Um estudo realizado entre 1976 e 1978 mostrou que a prevalência do consumo de psicofármacos no Estado de São Paulo, Brasil, foi de $12 \%$, sendo $7 \%$ no gênero masculino e $16 \%$ no gênero feminino, em um período de 12 meses. Os ansiolíticos, hipnóticos e sedativos representaram $86 \%$ do consumo total, sendo a maioria BZD; os $13 \%$ restantes foram neurolépticos, antidepressivos e anticomiciais. Houve um aumento do consumo de psicofármacos com o aumento da idade da população a partir da faixa dos 35 a 44 anos (16).

Outro estudo realizado no Estado do Rio de Janeiro, Brasil, constatou um consumo de $5 \%$ de psicofármacos nos 30 dias que antecederam o estudo, sendo $7 \%$ entre mulheres e $3 \%$ entre homens. Houve, também, um aumento do consumo destas drogas com o aumento da idade da população. Os an- siolíticos foram os mais consumidos, perfazendo um total de $85 \%$, seguidos pelos antiepiléticos, que contribuíram com $6 \%$ do consumo total (17).

$\mathrm{O}$ método da dose diária definida (DDD) indica a dose média de cada droga, prescrita por dia em adultos, de acordo com a indicação terapêutica principal. No Brasil, o consumo de BZD, utilizando-se este indicador, foi de 23,03 e 18,48 DDD/1 000 habitantes/dia, respectivamente, para os anos de 1988 e 1989 (18).

Um levantamento do consumo de substâncias ilícitas entre escolares adolescentes brasileiros foi desenvolvido, na Cidade de Ribeirão Preto, Brasil, no ano de 1990. No ano que antecedeu o estudo, $7 \%$ dos indivíduos consumiram psicofármacos, sendo que $2 \%$ relataram o uso no último mês (19). Percebe-se que a utilização de psicofármacos não é apenas uma questão clínica, mas revelase como um problema de saúde coletiva (20).

Dentre os psicofármacos, o cirurgiãodentista (CD) utiliza, terapeuticamente, na grande maioria dos casos, apenas as drogas ansiolíticas $(7,21,22)$. Entretanto, o CD realiza atendimento odontológico a indivíduos que podem estar utilizando psicofármacos (13). É importante considerar que o uso dos psicofármacos reduziu a ocupação dos leitos hospitalares, resgatando estas pessoas para o convívio social (14) e, conseqüentemente, para o atendimento odontológico ambulatorial.

Os psicofármacos apresentam reações adversas que têm implicações diretas no atendimento odontológico. Os antidepressivos, bem como os neurolépticos, podem causar xerostomia e hipotensão postural $(7,8)$. O uso prolongado de neurolépticos pode levar, em $40 \%$ dos indivíduos, à discinesia tardia, caracterizada por movimentos anormais iniciados na região orofacial (14). O diazepam e o lorazepam podem causar xerostomia, "gosto amargo", edema da língua, língua saburrosa e inflamação gengival. As reações adversas relacionadas à carbamazepina incluem xerostomia, glossite e estomatite. A fluoxetina, que é um antidepressivo, apresenta relatos de xerostomia, glossite, estomatite aftosa, edema e descoloração de língua. A fenitoína apresenta, como reação adversa, a hiperplasia gengival (23).

Algumas interações medicamentosas são de interesse para o CD. A utilização de anestésicos locais em pacientes que utilizam benzodiazepínicos pode potencializar o efeito cardiopressor dos anestésicos. Merece cautela, também, o uso de vasoconstritores adrenérgicos (associados a anestésicos locais) em pacientes que utilizam antidepressivos tricíclicos, devido à potencialização dos efeitos adrenérgicos (14, 24). O diazepam pode potencializar os efeitos tóxicos do anestésico local bupivacaína. A eritromicina, antimicrobiano utilizado na prática odontológica, pode aumentar o nível sérico e a ação farmacológica dos BZD, produzindo efeitos indesejáveis (25).

Levando em consideração as interfe rências diretas dos psicofármacos e o número reduzido de trabalhos sobre a utilização destes medicamentos no Brasil, principalmente entre usuários de serviços odontológicos, este trabalho objetiva estudar a utilização de psicofármacos entre os usuários das clínicas integradas de atenção primária da Faculdade de Odontologia da Universidade Federal de Minas Gerais (FO/UFMG). Além disso, o trabalho procurou verificar a influência das variáveis gênero, idade, ocupação, escolaridade e estado civil nesta utilização, assim como a freqüência de anotação em ficha clínica odontológica, pelos alunos, dos pacientes que utilizavam psicofármacos.

\section{MATERIAIS E MÉTODOS}

A população estudada compreendeu todos os pacientes seqüenciais, com idade a partir de 12 anos, atendidos nas clínicas integradas de atenção primária (CIAP) da FO/UFMG, no período de 2 a 27 de junho de 1997. As CIAP compreendem cinco disciplinas do currículo do curso de graduação em Odontologia, presentes em todo ciclo profissional, do quarto ao oitavo semestres. Assim, existem as CIAP I, II, III, IV e V, sendo que as CIAP III e IV são clínicas extramuros. $\mathrm{O}$ atendi- 
mento nas CIAP é gratuito, sendo realizado em convênio com o sistema único de saúde (SUS). O perfil do paciente da FO/UFMG é o seguinte: os pacientes são, em sua maioria, mulheres adultas, com menos de 8 anos de escolaridade, residentes na periferia de Belo Horizonte, sem acesso a tratamento no setor privado e de baixo nível econômico (26).

As listas dos pacientes das CIAP foram cedidas pelo centro de apoio, seleção e encaminhamento do usuário (CASEU) da FO/UFMG. A relação dos alunos matriculados por semestre do curso foi obtida na seção de ensino da FO/UFMG. Através destas listas foi realizado o controle das entrevistas.

As entrevistas exploraram os seguintes dados: 1) nome do aluno, 2) data e 3) CIAP; e, sobre o paciente, 4) nome; 5) sexo; 6) idade; 7) ocupação; 8) escolaridade; 9) estado civil; 10) utilização de medicamentos nas últimas 2 semanas (com nome do medicamento, dose, posologia, indicação, local de aquisição, tempo de utilização e anotação em ficha clínica odontológica); e 11) utilização de algum psicofármaco nos últimos 12 meses, com as mesmas especificações do item 10 . Os itens de 1 a 4 foram preenchidos antes da realização das entrevistas, de acordo com as listas do CASEU e da seção de ensino.

Os formulários de entrevista, prétestados, foram entregues a todos os alunos das CIAP, no início do atendimento clínico, sendo explicados os objetivos do estudo. Solicitou-se aos alunos que entrevistassem o(s) paciente(s) antes do atendimento do dia. Os pacientes receberam esclarecimentos quanto ao objetivo da pesquisa e ao caráter confidencial da mesma. Caso o paciente listado pelo CASEU tivesse tido alta ou tivesse abandonado o tratamento, este era substituído pelo novo paciente que o aluno estivesse atendendo. Em situação de ausência do paciente ou do aluno, por motivo de falta, foi dada uma nova chance de entrevista ao paciente na consulta seguinte (após 1 semana). Em caso de nova falta (do aluno ou do paciente), $o$ paciente era excluído da amostra. Desta forma, todos os pacientes tive- ram duas chances de entrevista. O trabalho dos entrevistadores foi monitorado por professores das CIAP, que possuíam um manual de instruções sobre a entrevista. Os pacientes atendidos em caráter emergencial não foram entrevistados.

A análise dos dados foi feita através do programa Epi Info, versão 6.0. A análise baseou-se na estimativa da prevalência, nos últimos 15 dias e 12 meses, do consumo global de psicofármacos, assim como na estimativa da prevalência, nos últimos 12 meses, segundo as variáveis sócio-demográficas gênero, idade, ocupação, escolaridade e estado civil. A análise estatística dos resultados utilizou o teste do qui-quadrado $\left(\chi^{2}\right)$ para diferença de proporções, considerando o nível de significância de 0,05 (27).

\section{RESULTADOS}

Foram entregues 424 formulários de entrevistas. Destes, 352 (83\%) foram devidamente preenchidos e devolvidos ao final do período de coleta de dados.

Em relação à distribuição de pacientes pelas clínicas, 104 (29\%) freqüentavam a CIAP I, $57(16 \%)$ a CIAP II, 46 $(13 \%)$ a CIAP III, 70 (20\%) a CIAP IV e $75(21 \%)$ a CIAP V. A idade dos pacientes variou de 12 a 61 anos, com média de 26,16 anos e desvio padrão igual a 10,89. As profissões mais relatadas pelos pacientes foram a de estudante $(30 \%)$ e doméstica/do lar/dona de casa (30\%); nenhuma das demais profissões alcançou valores relativos superiores a $4 \%$ do total. Do total de pacientes, 243 (69\%) eram mulheres e $109(31 \%)$ eram homens. Em termos de escolaridade, $61 \%$ citaram ensino fundamental incompleto; $15 \%$ citaram ensino médio incompleto; $9 \%$ disseram ter concluído o ensino fundamental (8 anos de estudo). Outros 9\% disseram ter concluído o ensino médio (pelo menos 11 anos de estudo); $1 \%$ dos pacientes não informaram este dado. Em relação ao estado civil, $63 \%$ dos pacientes relataram ser solteiros, $26 \%$ casados, $5 \%$ amasiados, $4 \%$ separados ou divorciados e $2 \%$ viúvos.
Em relação à utilização de algum medicamento nas 2 semanas que antecederam o estudo, 134 pacientes (38\%) responderam afirmativamente. $\mathrm{O}$ consumo de psicofármacos nas 2 semanas antes do estudo foi relatado por 13 indivíduos (4\%), com um total de 21 drogas psicotrópicas consumidas. Os ansiolíticos, antipsicóticos e antidepressivos representaram, cada um, $24 \%$ do total desta utilização. Os anticonvulsivantes e os outros psicofármacos somaram $14 \%$ do consumo global.

Os psicofármacos foram utilizados por 37 pacientes das CIAP (10\% do total dos pacientes) nos 12 meses antes do estudo, sendo que, ao todo, foram consumidos 52 medicamentos. Os ansiolíticos foram utilizados por 16 indivíduos, com consumo de 20 medicamentos (38\%). Os antidepressivos representaram o consumo de sete pacientes, que utilizaram sete medicamentos (13\%). Cinco pacientes relataram ter consumido antipsicóticos, perfazendo 10 drogas utilizadas (19\%). Três anticonvulsivantes $(6 \%)$ foram utilizados por dois pacientes das CIAP. O grupo das outras drogas psicotrópicas, que inclui os analgésicos opióides, foi consumido por 11 pacientes, com um total de 12 (23\%) medicamentos (tabela 1). Conforme os relatos, o diazepam foi o medicamento mais consumido, totalizando $17 \%$ do total de psicofármacos mencionados.

A grande maioria dos medicamentos foi prescrita pelo médico (85\%). Houve três relatos $(6 \%)$ de indicação por parte de amigo, vizinho ou parente, a mesma freqüência sendo encontrada para a auto-indicação (6\%). Houve uma referência (2\%) à indicação do "farmacêutico" e um paciente não soube informar a origem da indicação.

Em relação ao local de aquisição destas drogas, 22 pacientes (42\%) relataram ter adquirido a droga em farmácias privadas, 19 (37\%) no centro de saúde e cinco indivíduos (10\%) adquiriram a droga com amigo, vizinho ou parente. A aquisição do medicamento no hospital foi mencionada por cinco pessoas (10\%). Um indivíduo (2\%) não soube relatar o local da aquisição.

A idade de 23 anos foi escolhida como ponto de corte para análise da relação entre a idade e o consumo de 
TABELA 1. Distribuição dos grupos de psicofármacos mais utilizados por pacientes odontológicos nos 12 meses que antecederam o estudo, Belo Horizonte (MG), Brasil, 1997

\begin{tabular}{lcrc}
\hline \multirow{2}{*}{$\begin{array}{c}\text { Grupo de } \\
\text { psicofármacos }\end{array}$} & $\begin{array}{c}\text { Número de indivíduos } \\
\text { que consumiram }\end{array}$ & \multicolumn{2}{c}{ Número de medicamentos utilizados } \\
\cline { 3 - 4 } & 16 & $n$ & $\%$ \\
\hline Ansiolíticos & 7 & 7 & 38 \\
Antidepressivos & 5 & 10 & 13 \\
Antipsicóticos & 2 & 3 & 19 \\
Anticonvulsivantes & 11 & 12 & 6 \\
Outros & 37 & 52 & 100 \\
$\quad$ Total & 37 & & \\
\hline
\end{tabular}

${ }^{a}$ Alguns pacientes relataram uso de mais de um psicofármaco. psicofármacos, pois esta representa a mediana de idade da população estudada. No grupo com idade até 23 anos foi encontrado um consumo de psicofármacos, nos últimos 12 meses, igual a $4 \%$. No entanto, $17 \%$ dos usuários, com idade superior a 23 anos, relataram ter consumido algum psicofármaco neste mesmo período. Esta diferença é estatisticamente significativa $\left(\chi^{2}=16,22 ; P<0,01\right)$ (tabela 2).

Em relação ao sexo, o consumo de psicofármacos foi igual a $13 \%$ entre mulheres e $5 \%$ entre homens. $O$ consumo foi significativamente maior entre o sexo feminino $\left(\chi^{2}=4,20 ; P<\right.$ $0,05)$ (tabela 2).
Em relação à escolaridade, o universo foi dividido entre os indivíduos com até 8 anos de estudo (ensino fundamental completo) e indivíduos com mais de 8 anos de estudo. Não houve diferenças significativas no consumo destes dois grupos $\left(\chi^{2}=0,31 ; P>0,05\right)$ (tabela 2).

Para observar a relação entre o estado civil e o consumo de psicofárma$\cos$, foi feita uma divisão entre indivíduos que apresentavam união estável (casados, amasiados) e indivíduos com união considerada instável (solteiros, viúvos, separados). O consumo de drogas entre indivíduos com união estável $(16 \%)$ foi significativamente
TABELA 2. Uso de psicofármacos por pacientes odontológicos nos 12 meses anteriores ao estudo e sua relação com as variáveis sócio-demográficas, Belo Horizonte (MG), Brasil, 1997

\begin{tabular}{|c|c|c|c|}
\hline \multirow[b]{2}{*}{ Variável } & \multirow[b]{2}{*}{$n^{\mathrm{b}}$} & \multicolumn{2}{|c|}{ Indivíduos que utilizaram psicofármacos } \\
\hline & & $n$ & $\%$ \\
\hline \multicolumn{4}{|l|}{ Idade } \\
\hline Até 23 anos & 177 & 7 & 4 \\
\hline Mais do que 23 anos & 175 & 30 & 17 \\
\hline \multicolumn{4}{|l|}{ Sexo } \\
\hline Feminino & 243 & 31 & 13 \\
\hline Masculino & 109 & 6 & 5 \\
\hline \multicolumn{4}{|l|}{ Escolaridade $^{c}$} \\
\hline \multicolumn{4}{|l|}{ Ensino fundamental completo } \\
\hline (8 anos de estudo) & 247 & 27 & 11 \\
\hline Mais de 8 anos de estudo & 101 & 9 & 9 \\
\hline \multicolumn{4}{|l|}{ Estado civil } \\
\hline Casado, união/amasiado & 110 & 18 & 16 \\
\hline Solteiro, viúvo, separado & 242 & 19 & 8 \\
\hline \multicolumn{4}{|l|}{ Profissão } \\
\hline Dona de casa & 105 & 17 & 16 \\
\hline Outras & 247 & 20 & 8 \\
\hline
\end{tabular}

a Os totais variam devido à exclusão de informações não disponíveis.

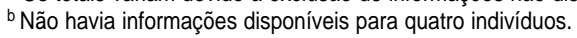

maior do que entre indivíduos do segundo grupo $(8 \%)\left(\chi^{2}=5,81 ; P<0,03\right)$ (tabela 2).

A profissão de dona de casa foi comparada com as demais, quanto ao consumo de psicofármacos. As donas de casa relataram um consumo igual a $16 \%$, significativamente maior do que o consumo de $8 \%$ dos outros indivíduos $\left(\chi^{2}=5,12 ; P<0,03\right)$ (tabela 2$)$.

A prevalência do consumo destes medicamentos foi maior na CIAP V, correspondendo a $20 \%$ dos pacientes. Em seguida tem-se a CIAP II, com um total de $14 \%$ de consumo, a CIAP IV, com uma prevalência de $8 \%$, e a CIAP III, com $6 \%$ de consumo entre os seus usuários. A CIAP I apresentou o menor valor percentual relacionado ao consumo de psicofármacos, com $5 \%$.

Dos alunos das CIAP, $40 \%$ mencionaram no formulário de entrevista que haviam anotado o uso das drogas psicotrópicas na ficha clínica do seu paciente; $36 \%$ não haviam anotado este dado, e $23 \%$ não informaram se a anotação foi realizada ou não.

\section{DISCUSSÃO E CONCLUSÕES}

O estudo do consumo de medicamentos entre os usuários dos serviços odontológicos tem dupla finalidade: uma epidemiológica, cuja importância refere-se aos poucos estudos no país (5, 28), à importância destes dados para diagnóstico e planejamento de serviços de saúde (4), às questões econômicas e sociais que envolvem o uso de medicamentos $(1,2,28)$ e à importância deste tema para a saúde coletiva no Brasil (20). A outra finalidade é clínica, fundamentada nas interferências diretas que os medicamentos exercem sobre a atenção odontológica $(6-14,21,23,24)$.

O consumo de psicofármacos entre usuários das clínicas foi de $4 \%$ e de $10 \%$, respectivamente, para os 15 dias e 12 meses anteriores ao estudo. Embora os dados não sejam comparáveis, por ser este um estudo de clientela, as prevalências encontradas têm valores próximos aos encontrados em trabalhos realizados no Brasil $(16,17)$.

Os ansiolíticos foram os mais consumidos por este grupo no período de 
12 meses, totalizando quase $40 \%$ do consumo global. Neste sentido, os estudos realizados no Brasil mostram uma tendência semelhante, porém mais acentuada $(16,17)$. A xerostomia, reação adversa importante, pode estar relacionada a maior risco no desenvolvimento da doença cárie dentária (29-32). Outra questão relevante são as conseqüências da diminuição da ansiedade sem uma necessidade real da utilização da medicação. A utilização de ansiolíticos pode levar à diminuição da participação ativa do indivíduo na sociedade (16) e, também, nos serviços de saúde, onde o usuário pode assumir uma posição ainda mais passiva (e mais paciente) em relação ao atendimento odontológico. Considerando como fundamental a responsabilidade do indivíduo em relação a sua saúde (33), o consumo de ansiolíticos pode ser considerado uma condição desfavorável para a saúde e, especificamente, para a saúde bucal.

Os antipsicóticos representaram 20\% do total de psicofármacos consumidos neste trabalho. Esta prevalência é superior àquela relatada em outros estudos realizados no Brasil (16, 17). A hipotensão ortostática e a discinesia tardia, reações adversas relacionadas ao uso de antipsicóticos (7, 8, 14, 34, 35), têm importância clínica direta no atendimento odontológico. A discinesia tardia também está relacionada ao uso de antidepressivos, inclusive aos fármacos mais recentemente utilizados (36).

$\mathrm{O}$ presente estudo encontrou um menor consumo de psicofármacos entre indivíduos mais jovens, resultado semelhante ao de outros estudos (16, 17, 19, 37). Da mesma forma, o maior consumo dos psicofármacos entre as mulheres, encontrado neste trabalho, está de acordo com estudos já realiza- dos $(16,17,38)$. Em relação a este último resultado, cabe colocar que a mulher subproletária recorre mais aos serviços de saúde (39). Os diversos problemas do seu cotidiano acarretam queixas (dor de cabeça, de coluna, problemas respiratórios) que os serviços de saúde ludibriam, muitas vezes, com a indicação de ansiolíticos como o diazepam (39). Entre as profissões mais citadas neste trabalho, a de dona de casa teve um consumo maior de psicofármacos em comparação às demais profissões. Estes últimos dados podem estar relacionados à já discutida relação entre o gênero feminino e maior consumo de psicofármacos. Dados coletados na Cidade de Belo Horizonte, no Brasil, mostram que as donas de casa representam a categoria que mais procura o centro de toxicomania devido à dependência de drogas, principalmente do álcool e dos benzodiazepínicos (40).

A escolaridade não esteve associada ao consumo de psicofármacos, resultado semelhante ao de Tancredi (16), mas diferente dos resultados de Almeida et al. (17). A relação encontrada entre união estável (casados, amasiados) e maior consumo de drogas psicotrópicas, em comparação com o consumo no grupo dos solteiros, viúvos e separados, não encontra resultados semelhantes nos estudos sobre consumo de psicofármacos no Brasil $(16,17)$.

Apesar dos efeitos diretos e indiretos do consumo de psicofármacos na atenção à saúde bucal dos usuários das CIAP da FO/UFMG, e apesar da significativa prevalência deste dado (10\%), parece que esta situação não é identificada como problema pelos futuros cirurgiões-dentistas. Apenas $40 \%$ dos estudantes das CIAP relataram haver anotado este dado na ficha clínica do paciente. No entanto, as ementas de todas as cinco disciplinas que envolvem as CIAP colocam como objetivo o diagnóstico e planejamento do atendimento odontológico (41). Acredita-se que, sem conhecimento dos aspectos biológicos (incluindo, dentre outros, a utilização de medicamentos), sociais e psicológicos do indivíduo, não é possível realizar um diagnóstico integral, tornando deficientes o planejamento e o atendimento.

Desta forma, o consumo de psicofármacos entre os usuários das CIAP da FO/UFMG apresenta um padrão semelhante ao de outros estudos já realizados no país, com algumas particularidades. Os acadêmicos que realizam o atendimento odontológico a estes indivíduos muitas vezes não identificam este consumo, o qual tem influências diretas sobre o tratamento. É necessária, então, uma reflexão sobre a importância da questão dos medicamentos na Odontologia. O ensino odontológico, que se mostrou falho neste aspecto, deve conscientizar-se destes dados para uma melhor formação do profissional de saúde. Espera-se que este trabalho possa permitir maiores discussões e estudos sobre a questão dos medicamentos (em particular dos psicofármacos) na área da saúde e, especificamente, na saúde bucal e no ensino odontológico.

Agradecimentos. Aos professores, alunos e pacientes das clínicas integradas de atenção primária da Faculdade de Odontologia da Universidade Federal de Minas Gerais, que colaboraram neste trabalho. Também ao Departamento de Farmácia Social e ao Centro de Estudos do Medicamento da Faculdade de Farmácia da Universidade Federal de Minas Gerais.

\section{REFERÊNCIAS}

1. Giovanni G. A questão dos medicamentos no Brasil. São Paulo: Polis; 1980.

2. Cordeiro H. A indústria da saúde no Brasil. 2 ed. Rio de Janeiro: Graal; 1985.
3. Béria JU. Consumo de medicamentos. Em: Victora CG, Barros FC. Epidemiologia da desigualdade. São Paulo: Hucitec; 1988. p. 137-147.
4. Loyola Filho AI. Investigação sobre utilização de serviços de saúde e consumo de medicamentos em um bairro periférico de Diamantina (MG) [Monografia]. Belo Horizonte, Mi- 
nas Gerais: Escola de Saúde de Minas Gerais; 1994.

5. Bricks LF, Leone C. Utilização de medicamentos por crianças atendidas em creches. Rev Saude Publica 1996;30(6):527-535.

6. Bloom FE. Transmissão neuro-humoral e o sistema nervoso central. Em: Gilman AG, ed. Goodman e Gilman - as bases farmacológicas da terapêutica. 8 ed. Rio de Janeiro: Guanabara-Koogan; 1991. p. 161-177.

7. Graeff FG. Drogas psicotrópicas e seu modo de ação. 2 ed. São Paulo: EPU; 1989.

8. Baldessarini RJ. Fármacos e o tratamento de distúrbios psiquiátricos. Em: Gilman AG, ed. Goodman e Gilman - as bases farmacológicas da terapêutica. 8 ed. Rio de Janeiro: Guanabara-Koogan; 1991. p. 253-287.

9. Van Der Bijl P. The benzodiazepines in dentistry: a review. Comp Contin Educ Dent 1992;13(1):46-50.

10. Henderson II BN, Triplett G, Cage TW. Anxiolitic therapy: oral and intravenous sedation. Dent Clin North Am1994;38(4):603-617.

11. Pereira LHMC, Ramos DLP, Crosato E. Ansiedade e dor em odontologia - enfoque psicofisiopatológico. Revista APCD 1995;49(4): 285-290.

12. Rall TW, Schleifer LS. Drogas eficazes no tratamento das epilepsias. Em: Gilman AG, ed. Goodman e Gilman — as bases farmacoló gicas da terapêutica. 8 ed. Rio de Janeiro: Guanabara Koogan; 1991. p. 288-304.

13. Sonis ST, Fazio RC, Fang L. Medicina oral. Rio de Janeiro: Guanabara Koogan; 1985.

14. Neidle EA, Kroeger DC, Yagela JA. Farmacologia e terapêutica para dentistas. 3 ed. Rio de Janeiro: Guanabara Koogan; 1991.

15. Pincus HA, Tanielian TL, Marcus SC, Olfson M, Zarin DA, Thompson J, et al. Prescribing trends in psychotropic medications. JAMA 1998;279(7):526-31.

16. Tancredi FB. Aspectos epidemiológicos do consumo de medicamentos psicotrópicos pela população de adultos do distrito de São Paulo [Dissertação]. São Paulo: Faculdade de Saúde Pública da Universidade de São Paulo; 1979.

17. Almeida LM, Coutinho ESF, Pepe VLE. Consumo de psicofármacos em uma região administrativa do Rio de Janeiro: a Ilha do Governador. Cadernos Saude Publica 1994;10(1): 5-16.
18. Nappo SA, Carlini EA. Benzodiazepínicos no Brasil: um perfil do consumo nos anos de 1988 e 1989. Em: Carlini EA. Medicamentos, drogas e saúde. São Paulo: Hucitec; 1995. p. 119-129.

19. Muza GM, Bettiol H, Muccillo G, Barbieri MA. Consumo de substâncias psicoativas por adolescentes escolares de Ribeirão Preto, SP (Brasil): I - Prevalência do consumo por sexo, idade, e tipo de substância. Rev Saude Publica 1997;31(1):21-29.

20. Franco RCS, Carvalho Neto JA, Khouri MA, Nunes MO, Santos Júnior JW, Marques Neto $\mathrm{J}$, et al. Consumo de medicamentos em um grupo populacional da área urbana de Salvador - BA. Rev Baiana Saude Publica 1986/1987;13/14(4/1):113-121.

21. Brasil, Divisão Nacional de Vigilância Sanitária de Medicamentos. Portaria 27. Diário Oficial da União 198624 outubro:16.370-16.371.

22. Brasil, Divisão Nacional de Vigilância Sanitária de Medicamentos. Portaria 28. Diário Oficial da União 198613 novembro:17.205-17.212.

23. Smith RG, Burtner AP. Oral side-effects of most frequently prescribed drugs. Spec Care Dent1994(3);14:96-102.

24. Wannmacher L. Anestésicos locais. Em: Wannmacher L, Ferreira MBC. Farmacologia clínica para dentistas. Rio de Janeiro: GuanabaraKoogan; 1995. p. 74-79.

25. Rocha MOC, Pedroso ERP, Fonseca JGM, Silva OA. Terapêutica clínica. Rio de Janeiro: Guanabara-Koogan; 1998.

26. Ferreira CN, Lopes AS, Silva JC, Pordeus IA, Paixão $\mathrm{HH}$. O perfil social do paciente da Faculdade de Odontologia da Universidade Federal de Minas Gerais. Em: Universidade Federal de Minas Gerais. Anais da IV Semana de Iniciação Científica da UFMG. Belo Horizonte: IV Semana de Iniciação Científica da UFMG; 1995. p. 88.

27. Dean AG, Dean JA, Coulombier D, Burton AH, Brendel KA, Smith DC, et al. Epi Info version 6: a word processing, data base, and statistics program for public health on IBM-compatible microcomputers. Atlanta: Centers for Disease Control and Prevention; 1995.

28. Fraser HS. Uso racional de los medicamentos esenciales. Foro Mundial de la Salud 1985;6: 74-78.

29. Thylstrup A, Fejerskov O. Cariologia clínica. 2 ed. São Paulo: Santos; 1995.
30. Opperman RV. Diagnóstico clínico e tratamento das doenças cárie e periodontal. Em: Mezzomo E. Reabilitação oral para o clínico. São Paulo: Santos; 1994. p. 7-59.

31. Mochel Filho JR. Algumas causas comumente não detectadas da cárie dental. Rev $\mathrm{ABO} \mathrm{Nac}$ 1998;6(1):37-43.

32. Lundgren M, Emilson CG, Osterberg T, Steen G, Birkhed D, Steen B. Dental caries and related factors in 88-92 years old. Acta Odont Scand 1997;55(5):282-291.

33. Capra F. O ponto de mutação, a ciência, a sociedade e a cultura emergente. São Paulo: Cultrix; 1989.

34. Consentino M, Leoni O, Rispoli L, Pellegrini C, Finavera L, Lecchini S, et al. A 1-year study of drug prescriptions and adverse drug reaction in psychiatric hospital practice. Pharmacoepidemiology Drug Safety 1996;5(6):377-384.

35. Scully C, Cawson RA. Medical problems in dentistry. 3 ed. Oxford: Wright; 1995.

36. D'arcy PF, Griffin JP. Interactions with drugs used in the treatment of depressive illness. Adverse Drug React Toxicol Rev 1995;14(4): 211-231.

37. Joukamaa M, Sohlman B, Lehtinen V. The prescription of psychotropic drugs in primary health care. Acta Psychiatr Scand 1995;92(5): 359-364.

38. Neuteu CI, Maxwell CJ. The benzodiazepine treadmill - does one prescription lead to more? Pharmacoepidemiology Drug Safety 1996;5(1):39-42.

39. Pereira WCCP. O adoecer psíquico do subproletariado. Belo Horizonte: Segrac; 1990.

40. Dependência atinge donas de casa. Estado de Minas 199826 de maio: 25 (Gerais/Saúde/ Urbanismo).

41. Universidade Federal de Minas Gerais, Faculdade de Odontologia, Colegiado de Graduação. Ementas das disciplinas do curso de graduação em Odontologia. Belo Horizonte: UFMG; 1998.

Manuscrito recebido em 4 de novembro de 1998. Aceito em versão revisada em 29 de junho de 1999. 
ABSTRACT In Brazil, psychotropics and other drugs are often indiscriminately overused. Nevertheless, there are few studies regarding the use of psychotropics, especially among dental patients. The purpose of this study was to assess the prevalence of psychotropic consumption among patients of the general primary care clinic of the Dentistry School at the Minas Gerais Federal University, in Brazil. To collect data, students working in the clinic interviewed all patients over 12 years of age seen at the clinic during June 1997 and asked them about their use of psychotropics during the preceding 2 -week and 12-month periods. The results showed that $4 \%$ of the patients had used psychotropic drugs in the 2 weeks before the study and that $10 \%$ of them had used psychotropics in the preceding 12 months. The drugs used most frequently in the 12-month period were anxiolytics (around $40 \%$ of total use). The median age of the patients was 23 years old. Persons under the median had used psychotropics less in comparison with older persons $(P<0.01)$. A significant association $(P<0.05)$ was found between using drugs and being female, and also between the use of drugs and being a housekeeper or a housewife $(P<0.03)$. Patients with a regular relationship (married or living together) used more psychotropics than patients who were single, widowed, or divorced $(P<0.03)$. There was no association between the use of drugs and the level of education. Even though information on the use of psychotropics is important for dental diagnosis and planning, only $40 \%$ of the students said they noted this information in their patients' charts. That fact suggests that dental student education may be lacking in this regard and that dentistry training should take into consideration the issue of patients' use of drugs. 